\title{
Rotura uterina intraparto: reporte de caso
}

\author{
Dr. Franklin José Espitia De La Hoz ${ }^{1}$
}

\section{RESUMEN}

La rotura uterina es un suceso infrecuente, pero de gravedad inconmensurable y la mayoría de las veces trágica. Suele acompañarse de catastróficas complicaciones, tanto para la madre como para el feto, como el shock hemorrágico, necesidad de histerectomía periparto, encefalopatía isquémica hipóxica, lesión cerebral permanente e incluso la muerte de ambos.

Palabras clave: Embarazo, Rotura uterina, Complicaciones, Hipoxia fetal, Cesárea.

\section{SUMMARY}

Uterine rupture is an infrequent event, but of immeasurable seriousness and most of the time tragic. It is usually accompanied by catastrophic complications, both for the mother and the fetus, such as hemorrhagic shock, the need for peripartum hysterectomy, hypoxic ischemic encephalopathy, permanent brain injury and even the death of both.

Keywords: Pregnancy, Uterine rupture, Complications, Fetal hypoxia, Cesarean section.

\section{INTRODUCCIÓN}

La rotura uterina en el embarazo es una complicación rara, pero catastrófica; se considera una emergencia obstétrica quirúrgica, ya que cursa con una alta incidencia de morbi-mortalidad fetal y materna. El pronóstico es malo para el binomio madre-hijo en la mayoría de los casos, en especial, si se produce en un útero sin cicatrices. En Estados Unidos se le adjudica el $5 \%$ de las muertes maternas, en todas las series descritas, sin embargo, la tasa de rotura uterina es, en general, de 1 en 1146 embarazos (0,07\%) (1-3). En

\footnotetext{
${ }^{1}$ Ginecología y Obstetricia, Universidad Militar Nueva Granada. Uroginecología / FUCS - Hospital de San José / Unicamp, Brasil. Especialista en Sexología Clínica. Máster en Sexología: Educación y asesoramiento sexual. Universidad de Alcalá de Henares. Servicio de Ginecología y Medicina Materno Fetal, Clínica La Sagrada Familia, Armenia, Quindío, Colombia.
}

países en vías de desarrollo, la muerte materna por estos casos asciende hasta un $50 \%$ (4) y la mortalidad fetal varía entre $14 \%$ y $100 \%(4,5)$.

La rotura uterina se presenta usualmente durante el trabajo de parto. Las causas incluyen antecedente de miomectomía, cesárea previa, embarazo múltiple, inadecuado uso de oxitócicos o prostaglandinas, parto instrumental complicado, polihidramnios, versión externa, etc. (6-8).

En fin, la rotura uterina en una mujer en trabajo de parto es una de las peores emergencias obstétricas que puede afrontar un obstetra, ya que no solo debe contar con la capacidad y preparación para abordarla, sino que el equipo debe demostrar su capacidad de respuesta ante un hecho tan grave y potencialmente letal para la gestante y su hijo. 


\section{CASO CLÍNICO}

Paciente de 24 años de edad, segundigesta (G2 P1 V1), procedente de área rural, ama de casa. Ingresó al servicio de maternidad manifestando inicio de actividad uterina irregular de 6 horas de evolución, con embarazo de 39,6 semanas (por ecografía del primer trimestre); adicionalmente refería disminución de movimientos fetales, lo cual la llevó a consultar.

Antecedentes: niega antecedentes patológicos, quirúrgicos, traumáticos y hospitalizaciones. Ginecoobstétricos: Menarquia: 12 años. Ciclos: 3-6/28. Inicio de vida sexual: 16 años. Edad primer parto: 21 años. Planificación: niega. Última menstruación: 18/03/2016. Parejas sexuales: 3. Infecciones de transmisión sexual: niega. Controles prenatales: 9, embarazo sin complicaciones. Grupo sanguíneo: A positivo. Transfusiones: niega. No presenta antecedentes familiares de importancia.

Estudios prenatales: (trae reportes)

03-05-2016: Toxoplasma IgG negativo. Antígeno de superficie para hepatitis B: negativo. Glicemia basal de $78 \mathrm{mg} / \mathrm{dl}$. VDRL: no reactivo. Urocultivo negativo.

Ecografías:

09-05-2016: Medición de la longitud cefalo-caudal para embarazo de 7,2 semanas \pm 3 días.

21-09-2016: Feto único, vivo, cefálico longitudinal, dorso derecho, fetocardia $141 \mathrm{lpm}$. Biometría para 27,3 semanas \pm 7 días. Placenta fundocorporal posterior grado II/IV. Índice de líquido amniótico (ILA): $12 \mathrm{~cm}$, cordón de 3 vasos. No circular de cordón. Peso fetal estimado: $1257 \pm 125$ gramos.

18-12-2016: Feto único, vivo, longitudinal, cefálico, dorso derecho. Fetocardia de 138 lpm. Peso fetal estimado: $3159 \pm 315$ gramos. ILA 8,4 cm. Placenta fundocorporal posterior grado II/IV. No malformaciones groseras. Embarazo de 39,2 semanas por biometría. Perfil biofísico de $8 / 8$.

Examen físico: TA: 108/72 mmHg; FC: 75 lpm; FR: 18 rpm; T: 36,9 grados; Peso: $57 \mathrm{Kg}$; Talla 1,56 m. Cabeza y cuello: mucosa oral húmeda, conjuntivas rosadas, no adenomegalias. Tórax: ruidos cardíacos rítmicos, murmullo vesicular presente, sin agregados. Abdomen: globoso por útero grávido, altura uterina: $33 \mathrm{~cm}$, feto único, vivo, longitudinal, cefálico, dorso derecho; frecuencia cardiaca fetal: 147 por minuto, no se evidencia dinámica uterina; puño percusión lumbar bilateral negativa. Genitourinario: genitales externos normoconfigurados. Tacto vaginal: cuello posterior, blando, corto, orificio cervical externo cerrado, estación cefálica flotante. Pelvis ginecoide. Extremidades: edema grado II, llenado capilar normal, pulsos distales presentes. Neurológico: sin déficit focalizado.

Se ingresa con diagnóstico de: embarazo de 39,6 semanas. Se le solicita monitoreo fetal, el cual se interpreta como no reactivo debido a la ausencia de aceleraciones y disminución de movimientos fetales. Se solicita perfil biofísico. ILA: $4,8 \mathrm{~cm}$.

Intervención terapéutica. Se decide hospitalizar para inducción de trabajo de parto por oligohidramnios. Se inicia goteo de oxitocina siguiendo protocolo institucional, el que se extiende hasta las 18 horas del primer día de la inducción, alcanzado una dosis máxima de $30 \mathrm{mU} / \mathrm{min}$, con la cual se logró una dinámica uterina de 2-3/10'/20-30' '/++, el monitoreo fetal se interpreta como reactivo por presentar tres aceleraciones con una línea de base de 153 lpm durante los 20 minutos El tacto vaginal muestra cambios: cuello dilatado $2 \mathrm{~cm}$, borramiento $60 \%$, estación -1 y membranas integras. Su evolución transcurre en la noche sin alteraciones. 
En la mañana del día siguiente de la hospitalización (dieciocho horas después del ingreso a la institución) se encuentra actividad uterina irregular, de corta duración y baja intensidad (1-3/10'/15-30'/ $/+$ ). Al tacto vaginal: cuello dilatado $4 \mathrm{~cm}$, borramiento $80 \%$, estación -1 y membranas integras. Se procede a la realización de amniotomía, obteniéndose escaso líquido grumoso, eutérmico, no fétido. No se observan cambios en el borramiento ni en la dilatación y se encuentra una variedad de posición occipitosacra (OS). No se observan alteraciones cardiotocográficas y se le inicia refuerzo con oxitocina (solución salina normal, $500 \mathrm{cc}$ más 3 UI de oxitocina) y se deja goteo a $2 \mathrm{mU} / \mathrm{min}$.

El partograma evoluciona lento, pero dentro de la curva de seguridad. A las 27 horas del ingreso, se encuentran dilatación y borramiento completo, estación 0 , OS persistente, por lo cual se realiza rotación manual de la cabeza fetal. A las dos horas se encuentra en expulsivo y estación +2 . Se traslada a sala de partos, precisando instrumentación con espátulas de Velasco (por considerarse expulsivo prolongado), maniobra que resulta fallida luego de dos intentos.

La mujer, durante la atención del parto, presentó cuadro de dolor abdominal agudo e intenso, cese súbito de las contracciones asociado a un retroceso en la progresión de la presentación y bradicardia fetal sostenida inferior a 72 lpm, compatible con sufrimiento fetal agudo; por lo cual fue sometida a operación cesárea de emergencia obteniéndose un recién nacido de sexo femenino de 3279 gramos, talla $51 \mathrm{~cm}$, con Apgar de 3 y 6 al minuto y cinco minutos respectivamente. Los gases de cordón fueron $\mathrm{pH} 7,32, \mathrm{PaO}_{2} 114, \mathrm{PaCO}_{2} 45, \mathrm{BE}-2,4$. El neonato fue dado de alta luego de una semana en la unidad de cuidados intensivos neonatales.

En el transcurso de la operación cesárea, se encontró feto y placenta en la cavidad abdominal, por delante del útero, con un hemoperitoneo de aproximadamente 1200 cc. El útero mostró una ruptura segmentaria

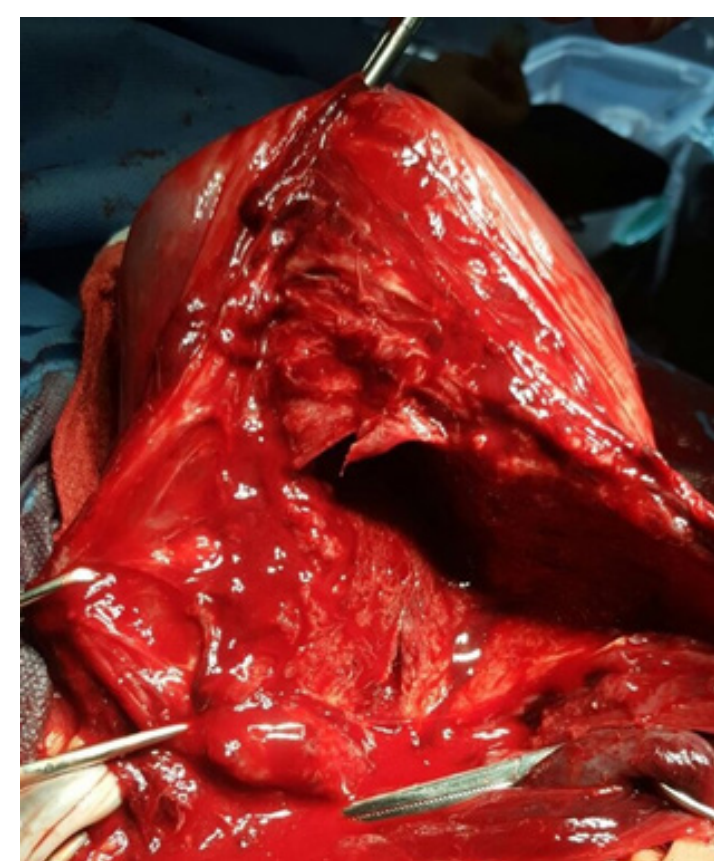

Figura 1. Ruptura segmentaria con compromiso de la cara lateral derecha del útero.

con compromiso de la cara lateral derecha (figura 1). Se realizó sutura uterina en doble capa a nivel del defecto, con poliglactina $910 \# 1$, sin inconvenientes. En el intraoperatorio se le transfundieron 3 unidades de glóbulos rojos empaquetados y otra unidad se transfundió después de la cirugía.

El postoperatorio puerperal transcurrió sin complicaciones. La hemoglobina posoperatoria fue de $10,8 \mathrm{~g} / \mathrm{dl}$ y la paciente fue dada de alta 6 días después de la cirugía.

\section{DISCUSIÓN}

La rotura uterina en trabajo de parto es una de las peores emergencias obstétricas en el ejercicio profesional de un obstetra, debido a que están en peligro la vida de la madre y el feto, pero asimismo está en juego su idoneidad como especialista. 
En esta mujer se presentan varios factores de riesgo descritos como potenciales predisponentes para la rotura uterina: trabajo de parto prolongado, distocia de presentación, parto instrumentado y rotación de la variedad de posición (6-8). El resultado exitoso de la actuación en esta paciente se debe a la intervención y tratamiento oportunos, como también a contar con los elementos necesarios (disponibilidad de sangre, unidad neonatal, grupo de anestesia, quirófano disponible, etc.), para ofrecerle una adecuada atención, además de la oportuna intervención quirúrgica; lo cual ha sido establecido anteriormente por otros autores (9).

No se puede establecer, con el presente caso, una contundente incidencia, pero se puede hipotetizar que en este centro estaría alrededor de 1 por cada 1764 partos, lo cual es menor a lo reportado por otras publicaciones (10-13).

El diagnóstico de rotura uterina no suele ser el florido caso de libro, puesto que los clásicos signos de rotura solo se presentan en un tercio de los casos $(14,15)$, lo que obliga a estar más atentos a los cambios generales en la parturienta; sin embargo, la frecuencia cardíaca fetal disminuida, suele ser la anormalidad constante $(16,17)$.

La puntual intervención en la realización de la cesárea de inmediato en esta mujer, alentó a no terminar en histerectomía ni a la prolongación de la agresión hipóxica perinatal, facilitando los buenos resultados maternos y neonatales. El manejo quirúrgico de la rotura uterina depende del tipo, lugar de la rotura, gravedad de la hemorragia, así como de los deseos de la mujer respecto de la conservación de la fertilidad futura.

La conveniencia de contar con unidades obstétricas equipadas de forma idónea con adecuada infraestructura humana y técnica, permitieron que esta rotura uterina se pudiera enfrentar sin peores complicaciones, lo cual se presume al ver que el trabajo de parto fue vigilado y controlado de forma adecuada, a pesar de la prolongación de la fase activa y del expulsivo, con la intervención quirúrgica obstétrica realizada a tiempo.

\section{CONCLUSIONES}

La rotura uterina es un evento impredecible y, aunque usualmente ocurre durante el trabajo de parto en mujeres con cicatriz uterina previa, en este caso se documentó que, a veces, puede presentarse en un útero intacto.

Este caso enseña que las maniobras realizadas, muchas de ellas rutinarias en la mayoría de las unidades obstétricas, deben considerarse y recordarse como factores de riesgo de rotura uterina, incluso en úteros sin cicatrices.

Finalmente, un dolor abdominal agudo y severo, en una mujer en trabajo de parto, que se asocia a bradicardia sostenida con ausencia de actividad uterina, debe ser abordado y manejado con atenta seriedad.

Los autores declaran no tener conflicto de intereses.

\section{REFERENCIAS}

1. Ahmadi S, Nouira M, Bibi M, Boughuizane S, Saidi H, Chaib A, et al. Rupture utérine sur utérus sain gravide. A propos de 28 cas [Uterine rupture of the unscarred uterus. About 28 cases]. Gynecol Obstet Fertil. 2003; 31(9):713-717. French.

2. Cavanagh D, Membery J, Macleod G. Rupture of the gravid uterus: An appraisal. Obstet Gynecol. 1965; 26(2):157-164.

3. Van der Merwe JV, Ombelet WU. Rupture of the uterus: a changing picture. Arch Gynecol. 1987; 240(3):159171.

4. Adanu RM, Obed SA. Ruptured uterus: a seven-year review of cases from Accra, Ghana. J Obstet Gynaecol Can. 2003; 25(3):225-230. 
5. Semchyshyn S, Gerulath AH, Strickler RC. Infant survival following uterine rupture and complete abruptio placentae. Obstet Gynecol. 1977; 50(1 Suppl):74s-75s.

6. AL Salem MH, Makhseed M, Ahmed MA, Gupta M. Rupture of the gravid uterus: experience of the maternity hospital, Kuwait. Med Principles Pract. 2000; 9:97-105

7. Ames RPM: Rupture of the uterus. Am J Obstet Gynecol 1881; 14:361-395.

8. Lydon-Rochelle M, Holt VL, Easterling TR, Martin DP. Risk of uterine rupture during labor among women with a prior cesarean delivery. N Engl J Med. 2001; 345(1):3-8.

9. Bernstine R. Rotura uterina. En: Iffy L, Kaminetzky $\mathrm{H}$, editores. Obstetricia y Perinatología. Principos y práctica. Volumen 2. Buenos Aires: Editorial Médica Panamericana; 1986; p. 416-418.

10. Groen GP. Uterine rupture in rural Nigeria: review of 144 cases. Obstet Gynecol. 1974; 44(5):682-687.

11. Yussman MA, Haynes DM. Rupture of the gravid uterus. A 12-year study. Obstet Gynecol. 1970; 36(1):115-120.

12. Akasheh F. Rupture of the uterus. Analysis of 104 cases of rupture. Am J Obstet Gynecol. 1968; 101(3):406408.
13. Zeller RE, Schwarz RH, Emich JP Jr. Uterine rupture: an analysis of 43 cases. Obstet Gynecol. 1966; 27(6):859-862.

14. Turner MJ, Robson MS, MacDonald D, Stronge JM. Successful outcome after antepartum expulsion of placenta and fetus into the abdominal cavity; a case report. Eur J Obstet Gynecol Reprod Biol. 1989; 33(2):187-188.

15. Schrinsky DC, Benson RC. Rupture of the pregnant uterus: a review. Obstet Gynecol Surv. 1978; 33(4):217232.

16. Leung AS, Leung EK, Paul RH. Uterine rupture after previous cesarean delivery: maternal and fetal consequences. Am J Obstet Gynecol. 1993; 169(4):945950.

17. Turner MJ, Flannelly GM, Wingfield M, Rasmussen MJ, Ryan R, Cullen S, et al. The miscarriage clinic: an audit of the first year. Br J Obstet Gynaecol. 1991; 98(3):306-308 